\title{
Microbiological spectrum and antimicrobial susceptibility patterns of various isolates from endotracheal tube aspirates in a tertiary care hospital, Hyderabad, Telangana
}

\author{
A. Swati1 ${ }^{1, *}$, K. Yamini' ${ }^{2}$ R. V. Rajkumar ${ }^{3}$ \\ ${ }^{1,2}$ Assistant Professor, ${ }^{3}$ Professor \& HOD, Dept. of Microbiology, Kamineni Academy of Medical Sciences \& Research Centre, \\ Hyderabad, Telangana, India \\ *Corresponding Author: \\ Email: drswathi.gurajala@gmail.com
}

\begin{abstract}
Introduction: Endotracheal aspirates ( ET) are relatively simple ,easy to collect at the bedside and are used to evaluate the causes of lower respiratory tract infections in ventilated patients.Several studies reported multidrug resistant bacteria like Klebsiella, Pseudomonas and Acinetobacter spp,gram positive bacteria like S.aureus, S.pneumoniae ,Coagulase negative staphylococcus (CONS) and fungi like Candida spp in ET cultures. Even though Quantitation of the bacteria isolated from ET aspirates has been proposed as a criterion to distinguish colonization from true infection, only few studies have been done .The main aim of the present study was to evaluate the microbiological spectrum and antimicrobial susceptibility pattern of isolates from ET aspirates.

Materials and Methods: ET aspirates were processed employing quantitative cultures. Growth of $\geq 10^{5} \mathrm{cfu} / \mathrm{ml}$ was considered to be significant.Organism Identification and Antimicrobial susceptibility testing was performed according to standard guidelines. The gram negative isolates were further tested for ESBL/carbapenamase production and gram positive as methicillin sensitive / methicillin resistance.

Results: Culture positivity was $67 \%$. Acinetobacter spp was the most common isolate (46\%) followed by Klebsiella spp (26\%) and Pseudomonas spp (17\%), E.coli (5\%), S.aureus (2\%), CONS (2\%) and Candida (2\%). Acinetobacter spp were extremely drug resistant. ESBL was produced by $38 \%$ and $64 \%$ of E. coli and Klebsiella spp.

Out of the 15, 40\% of the Pseudomonas spp and of 127 isolates, 63.7\% of Acinetobacter spp were positive for carbapenamase production respectively .Out of the 7, $6(86 \%)$ were methicillin resistant Staphylococcus aureus.5 (83\%) of CONS (n=6) were methicillin resistant.

Conclusion: We conclude that ET microscopic examination and quantitative cultures can be supportive in earlier prediction of ventilator-associated pneumonia.
\end{abstract}

Keywords: Endotracheal aspirates, Microbiological spectrum, Quantitative cultures, Multidrug resistant organisms, infection control.

\section{Introduction}

Investigation of the bacterial causes of lower respiratory tract infection is hindered by access to the site of infection. Endotracheal (ET) aspiration helps in the evaluation for the occurrence of the febrile episodes in the mechanically ventilated patients to assess the patients risk to develop Ventilator associated pneumonia (VAP) or Hospital acquired pneumonia (HAP). ${ }^{1-3}$ The procedure is easily performed at the bedside, relatively simple, minimally invasive and inexpensive, has a proven acceptable accuracy and requires minimal investment for the training of health professionals. $^{4}$

Several studies demonstrated the ET culture as an additive diagnostic tool along with the routine tests in detection of plausible pneumonia pathogen. ${ }^{5-7}$

Internationally several studies done on ET aspirates reported predominantly gram negative organisms including Klebsiella spp, Pseudomonas spp, Enterobacter spp, Acinetobacter spp.and gram positive bacteria like Staphylococcus aureus. ${ }^{89}$ In India studies have been carried out to evaluate the organisms isolated from the ET aspirate and demonstrated the significant isolation of gram negative organisms including Klebsiella spp, Acinetobacter spp, Pseudomonas spp. ${ }^{10-}$ 14

Bacterial resistance to commonly used antimicrobial agents is again a commonly encountered problem in the Intensive care units. Several studies from Delhi ,Karnataka have demonstrated the isolation of multidrug resistant gram negative organisms from the ventilated patients. ${ }^{12-14}$ A study conducted in neighbouring state Andhra Pradesh demonstrated the isolation of the multi drug resistant Klebsiella, Pseudomonas and Acinetobacter spp,gram positive bacteria like S.aureus, S.pneumoniae, Coagulase negative staphylococcus spp (CONS) and fungi like Candida spp. ${ }^{15}$

Even though Quantitation of the bacteria isolated from ET aspirates has been proposed as a criterion to distinguish colonization from true infection, only few studies have been done by employing the quantitative cultures. ${ }^{16-18}$

With this background, the main aim of the present study was to evaluate the microbiological spectrum, importance of quantitative cultures and antimicrobial 
susceptibility pattern of isolates from ET aspirate in the Eastern part of the Hyderabad.

\section{Materials and Methods}

A retrospective study was conducted in the Dept. of Microbiology of our tertiary care centre for ET aspirates received between Jan -Dec 2017. A total 470 ET samples were received in Microbiology laboratory and processed using standard microbiological procedures like microscopy, culture and antimicrobial susceptibility testing.

\section{Microbiological processing}

The endotracheal aspirates sent to the lab were processed immediately. The samples were first subjected to Gram's staining and then quantitative cultures were performed. Endotracheal aspirate samples were considered valid for culture if $<10$ squamous epithelial cells and $>25$ neutrophils were present.

The sample rejection criteria were i) if there were $>10$ squamous epithelial cells/LPF in the sample ii) any duplicate specimens received on the same day unless the initial sample was inappropriate for culture according to microscopic evaluation and iii) repeat cultures at intervals of less than every 48 hours. ${ }^{18}$

All samples were plated on Blood agar (BA), Chocolate agar (CA), CPSE agar and Saboraud's dextrose agar (SDA). ET aspirate was serially diluted in sterile normal saline as $1 / 10,1 / 100,1 / 1000$ and $0.01 \mathrm{ml}$ of 1/1000 dilution was inoculated on 5\% sheep blood agar. After incubation at $37 \mathrm{C}$ for $24 \mathrm{~h}$, colony count was done and expressed as $\mathrm{CFU} / \mathrm{ml}$. The number of $\mathrm{CFU} / \mathrm{ml}$ is equal to number of colonies on agar plate $\times$ dilution factor $x$ inoculation factor. Therefore presence of even a single colony on the blood agar after inoculating $0.01 \mathrm{ml}$ of $1 / 1000$ times diluted ET aspirate was interpreted as more than $10^{5} \mathrm{CFU} / \mathrm{ml} .{ }^{18}$ (Fig. 1a). SDA plates incubated at room temperature were checked for any growth up to one week.

Quantitative culture threshold of $\geq 10^{5} \mathrm{cfu} / \mathrm{ml}$ was considered to be significant. Growth of any organism below the threshold was assumed to be due to colonization or contamination.

A detailed biochemical testing was done to identify any significant growth.

Antimicrobial susceptibility testing of the isolated organisms was performed by the disk diffusion technique by Kirby Bauer method.

Antimicrobials tested for gram negative isolates were amikacin $(30 \mu \mathrm{g})$, gentamicin $(10 \mu \mathrm{g})$, cefotaxime, ceftazidime $(30 \mu \mathrm{g})$, ciprofloxacin $(5 \mu \mathrm{g})$, piperacillintazobactam $(75+10 \mu \mathrm{g})$, cefoperazone-sulbactam $(75+30$ $\mu \mathrm{g})$, imipenem $(10 \mu \mathrm{g})$, meropenem $(10 \mu \mathrm{g})$. Colistin Ezy MIC ${ }^{\mathrm{TM}}$ Strip $(0.016-256 \mathrm{mcg} / \mathrm{ml})$ (Himedia) was used to determine the minimum inhibitory concentration (MIC).

Antimicrobials tested for gram positive isolates were amikacin $(30 \mu \mathrm{g})$, gentamicin $(10 \mu \mathrm{g})$, cefotaxime, ceftazidime(30 $\quad \mu \quad \mathrm{g}), \quad \operatorname{ciprofloxacin}(5 \quad \mu \quad \mathrm{g})$, erythromycin(15 $\mu \mathrm{g})$ linezolid(30 $\mu \mathrm{g})$, teicoplanin(30 $\mu \mathrm{g})$ discs. Vancomycin Ezy MIC ${ }^{\mathrm{TM}}$ Strip (0.016-256 $\mathrm{mcg} / \mathrm{ml}$ ) (Himedia) was used to determine the minimum inhibitory concentration (MIC).

Interpretation of the zone diameters and breakpoints of MICs was done as per clinical laboratory and standards institute (CLSI) guidelines 2017. ${ }^{19}$

For Extended spectrum beta lactamase (ESBL) detection, disc diffusion method was performed on muller hinton agar(MHA) with ceftazidime $(30 \mu \mathrm{g})$ and ceftazidime- clavulanic acid $(30 / 10 \mu \mathrm{g})$. A $\geq 5 \mathrm{~mm}$ increase in zone diameter for either antimicrobial agent tested in combination with clavulanate vs zone diameter of the agent when tested alone was identified as ESBL producers. ATCC Klebsiella 700603 and ATCC E.coli 25922 were used as QC strains.

For Metallo beta lactamases (MBL) detection, Modified hodge test (MHT) was performed. A $0.5 \mathrm{Mc}$ Farland standard suspension of ATCC E.coli 25922 was prepared and inoculated as routine lawn culture on the MHA. The plates are allowed to dry for 3-10 mins.Appropriate numbers of meropenam $(10 \mu \mathrm{g})$ discs are placed. The test organism was inoculated from the edge of the disc. The streak should be about 24$25 \mathrm{~mm}$ length and incubated for $16-20 \mathrm{hrs}$ at 37C.Following incubation the MHA plates were examined for enhanced growth around the test or QC organism streak at the intersection of the streak and ZOI. Any evidence of enhanced growth was considered positive for carbapenamase production and no enhancement in growth are considered negative for carbapenamase production. Klebsiella ATCC BAA-1705 and Klebsiella ATCC BAA-1706 were taken as positive and negative controls for MHT.

For detection of methicillin resistance in Staphylococcus spp, $30 \mu \mathrm{g}$ cefoxitin disc was placed on the lawn culture of the test organism on MHA .The plate was incubated for 16-18hrs( if Staphylococcus aureus ) and for 24hrs( if CONS). For Staphylococcus aureus, zone of inhibition (ZOI) $<21 \mathrm{~mm}$ was considered as resistant (mec A positive) and $\geq 22 \mathrm{~mm}$ was considered as sensitive (mecA negative). For CONS, ZOI $\leq 24 \mathrm{~mm}$ was considered as resistant (mec A positive) and $\geq 25 \mathrm{~mm}$ as sensitive (mec A negative). S.aureus ATCC 43300 and S.aureus ATCC 25923 were taken as positive and negative controls. ${ }^{19}$

\section{Results}

A total of 470 ET aspirates were received in the Department of Microbiology in 1 year.

Out of this, 314 samples $(67 \%)$ were culture positive with significant colony counts $\geq 10{ }^{5} \mathrm{cfu} / \mathrm{ml}$ (Fig. 1b). Gram negative bacteria were the predominant isolate $(n=294)$ followed by Gram positive cocci $(n=13)$ and Candida $(\mathrm{n}=7)$.

Among the GNB, Acinetobacter spp was the most common isolate $(46 \%)$ followed by Klebsiella spp (26\%) and Pseudomonas spp (17\%). (Fig 2) 
Less frequently encountered organisms were E.coli (5\%), S.aureus (2\%), CONS (2\%) and Candida (2\%). Most of the isolates are from RICU (72\%) followed by medical and surgical ICUs. (Table 1)

The associated risk factors are shown in the Fig. 3 The antimicrobial susceptibility pattern of gram negative and gram positive organisms is shown in Fig. $4 \mathrm{a} \& 4 \mathrm{~b}$.

Among the 143 isolates of Acinetobacter spp, 92-95\% was resistant to fluororquinolones, aminiglycosides, $3^{\text {rd }}$ generation cephalosporins and betalactam +betalactam inhibitor combinations. About $89 \%$ of the isolates were resistant to carbapenams.

Among 81 isolates of Klebsiella spp, $64-79 \%$ isolates were resistant to fluororquinolones, aminiglycosides, $3^{\text {rd }}$ generation cephalosporins and betalactam +betalactam inhibitor combinations. About $52 \%$ of the isolates were resistant to carbapenams.

Pseudomonas spp and E.coli showed a variable resistance to all the tested antibiotics.

None of the isolates were resistant to polymixins.

ESBL was produced by $38 \%$ and $64 \%$ of $E$. coli and Klebsiella spp respectively.

Out of the 15 isolates of the Pseudomonas spp and 127 isolates of Acinetobacter spp resistant to carbapenams, $6(40 \%)$ and $81 \quad(63.7 \%)$ were positive for carbapenamase production respectively by Modified hodge test.

Out of the 7 strains of Staphylococcus aureus, 6 (86\%) were methicillin resistant.

$5(83 \%)$ of $\operatorname{CONS}(\mathrm{n}=6)$ were methicillin resistant.

However, all the strains were sensitive to Vancomycin.

\section{Discussion}

Ventilator associated pneumonia (VAP) or severe community acquired pneumonia (CAP) is associated with high mortality and morbidity rates. Thus, early and accurate diagnosis and appropriate empirical antibiotic treatment are important outcome variables. However, it is often difficult to obtain specimens from the lower respiratory tract without contamination by the colonizing oropharyngeal bacteria. Generally qualitative cultures are performed and the results are invariably often difficult to interpret. Since these cultures are unable to distinguish between pathogens and colonizing bacteria, the concept of quantitative culture was developed based on bronchoalveolar lavage (BAL), protected specimen brush (PSB), blinded protected telescoping catheter, and ET cultures. ${ }^{14} \mathrm{Few}$ studies included comparative evaluations of the accuracy of quantitative EA cultures for the diagnosis of VAP versus other diagnostic methods, such as, autopsy specimen, BAL, or PSB culture, or clinical methods. ${ }^{15,16}$ Invasive procedures eg. Bronchoscopy PSB are usually performed only in the later stages of VAP, may rarely lead to cardiac arrhythmias, hypoxemia, or bronchospasm. ${ }^{20}$ So, there is a need for a non-invasive technique which can be performed early in patients suspected to have developed VAP. Quantitative EA cultures are straightforward, easily performed at bed side, cheap and non-invasive.

In our study there is increased emergence of multidrug resistant (MDR) Acinetobacter spps, Klebsiella spp and Pseudomonas spp as potential pathogens from endotracheal aspirates especially from ICUs. Several studies have also reported the same bacterial flora. ${ }^{9-14}$

Comorbid conditions like Type-II Diabetes mellitus (DM), Hypertension (HTN), Chronic obstructive pulmonary disease (COPD) and Alcohol were documented in our study population. Similar observations were documented by Lakshmi et al and Dey et al. ${ }^{16,21}$

Among our cases, we isolated 4 Candida albicans $(1.2 \%)$ and 3 Candida non-albicans $(0.9 \%)$ with colony count $<10^{4} \mathrm{cfu} / \mathrm{ml}$ which determines that Candida was tracheal colonizer. Hamet et al reported that the association between MDR bacteria and Candida colonization was more likely due to shared risk factors rather than causal association. ${ }^{22}$

In the present study, both the prior antibiotic therapy and ICU length of stay could be important factors associated with the isolation of MDR organisms from patients who are intubated. Trouillet et al also suggested that previous antibiotic use and previous use of broad-spectrum antibiotics were associated with increased risk of developing pneumonia due to MDR organisms. ${ }^{23}$

In our study, $89 \%$ Acinetobacter species showed extreme drug resistance (XDR) which is in concordance with Dey et al. ${ }^{21}$

ESBLs and MBLs are of increasing clinical concern but have to be documented for epidemiological and infection control point of view. The spread of these organisms in nosocomial and community-acquired enterobacteria is an important challenge for clinicians as the therapeutic options for these organisms are limited. The epidemiology of these infections is complex and combines the expansion of mobile genetic elements with clonal spread. Infections caused by ESBL and carbapenamase producers are associated with increased mortality, length of stay and increased cost. $^{24}$

In our study ESBL producers were common among Enterobacteriaceae members like Klebsiella pneumonia 52/81(64\%) and Escherichia coli 6/16(38\%).Similar results have been reported by Ankita et al and Dey et al. ${ }^{14,21}$

In our study, 6 (40\%) of Pseudomonas spps were carbapenamase producing strains. Similar observations were made by Dey et al $(50 \%)^{21}$ and Goel et al $(47.06 \%){ }^{25}$

Out of 7 isolates of Staph.aureus, $6(86 \%)$ were methicillin-resistant Staphylococcus aureus (MRSA). This observation cannot be considered as high percentage as the number of isolates are few $(n=7)$. 
Prevalence of MRSA among ET aspirates was about $18.15 \%$ in a study by Veena Krishnamurthy et al. ${ }^{26}$

Hence, we recommend combined clinical, microbiological and infection control strategies which include proper diagnosis, appropriate specimen, quantitative cultures, and appropriate antimicrobial stewardship can lead to proper patient management.

Robust infection control measures are always important to prevent the spread of infection. The best approach to manage the colonization of ET tubes will be adaptation of proper infection control measures. Appropriate training of health care professionals regarding ventilator bundle care seems to be effective in reducing $\mathrm{VAP}^{27}$

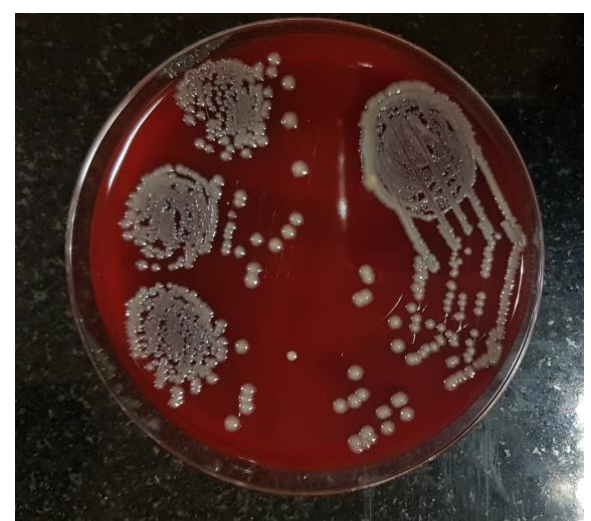

Fig. 1a: Quantitative cultures of ET aspirates on Blood agar and CPSE agar
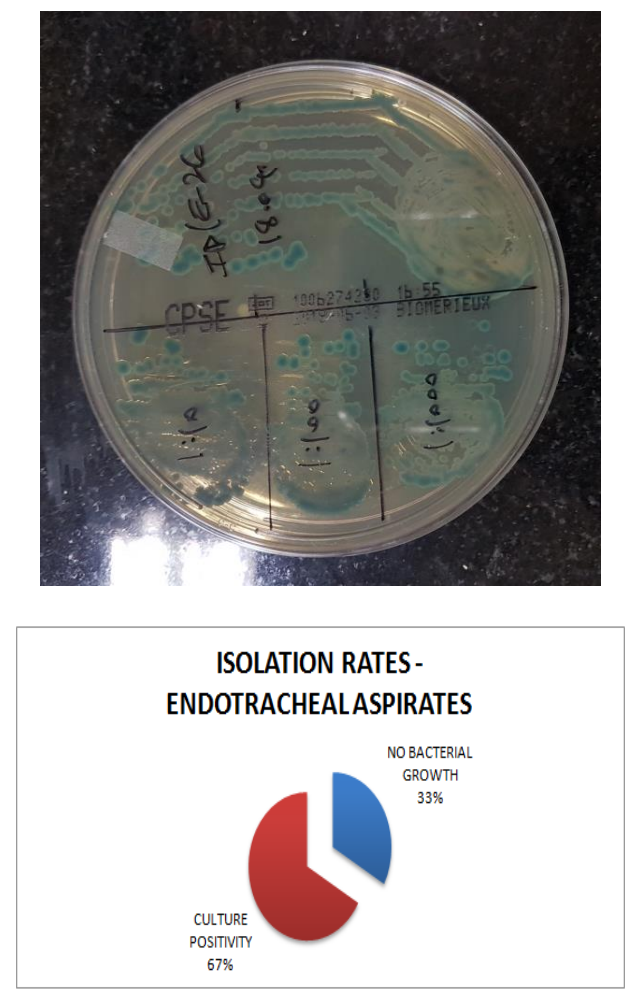

Fig. 1b: Isolation rates endotracheal aspirates

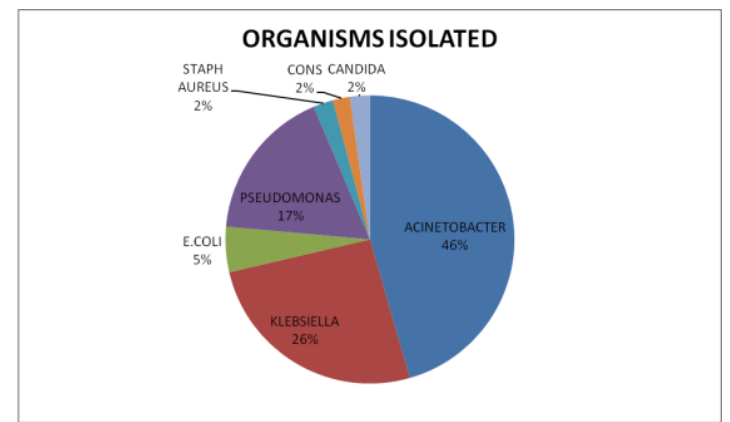

Fig. 2: Organisms Isolated

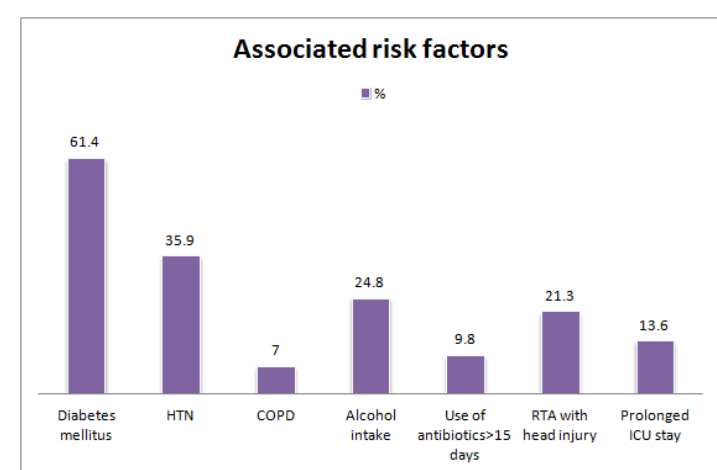

Fig. 3: Associated risk factors

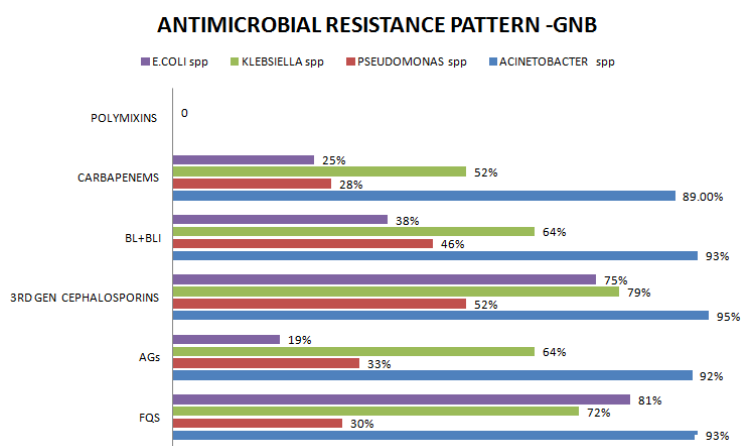

ANTIMICROBIAL RESISTANCE PATTERN- GPC

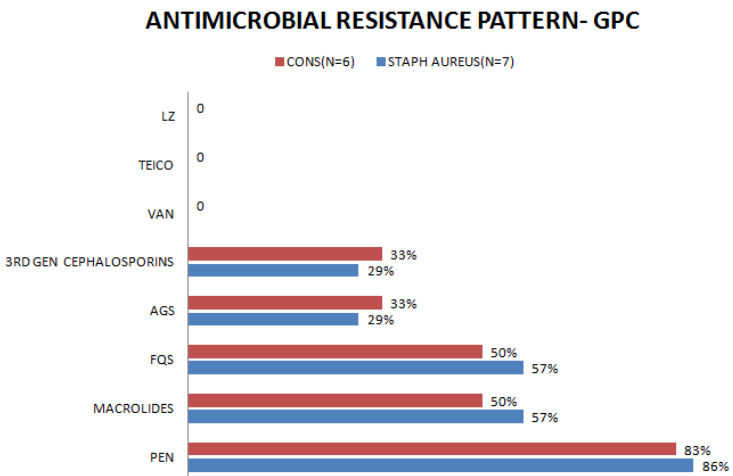

Fig. 4a \& 4b 
Table 1: Wardwise isolation of different organisms

\begin{tabular}{|l|c|c|c|c|c|c|c|c|}
\hline $\begin{array}{l}\text { Ward } \\
\text { Wise }\end{array}$ & Acinetobacter & Klebsiella & E. Coli & Pseudomonas & $\begin{array}{c}\text { Staph } \\
\text { Aureus }\end{array}$ & Cons & Candida & $\begin{array}{c}\text { Total No. of } \\
\text { isolates }\end{array}$ \\
\hline RICU & 107 & 56 & 12 & 337 & 5 & 4 & 6 & 227 \\
\hline $\begin{array}{l}\text { Medical } \\
\text { ICUs }\end{array}$ & 10 & 7 & 1 & 1 & 2 & 1 & 1 & 23 \\
\hline $\begin{array}{l}\text { Surgical } \\
\text { ICUs }\end{array}$ & 14 & 9 & 3 & 8 & 0 & 0 & 0 & 34 \\
\hline PICU & 2 & 0 & 0 & 2 & 0 & 0 & 0 & 4 \\
\hline OBG & 0 & 0 & 0 & 2 & 0 & 0 & 0 & 2 \\
\hline Ward & 10 & 9 & 0 & 4 & 0 & 1 & 0 & 24 \\
\hline & & & & & & & & 314 \\
\hline
\end{tabular}

\section{Conclusion}

We conclude that endotracheal aspirate microscopic examination and quantitative cultures can be supportive in objective diagnosis of ventilatorassociated pneumonia with an added advantage of earlier prediction.

\section{References}

1. Hoque L, Kamal SMM, Z Ahmed. Isolation and identification and the antimicrobial sensitivity pattern of bacterial isolates from the tracheal aspirates of ICU patients of central Dhaka, Bangladesh. IJRANSS. Dec 2013;1(7):11-6.

2. Ashworth M, Latinovic R, Charlton J, Cox K, Rowlands G, Gulliford M. Why has antibiotic prescribing for respiratory illness declined in primary care?

Alongitudinal study using the general practice research database. J Public Health. 2004 ;26:268-74.

3. Cook D, Mandell L. Endotracheal Aspiration in the Diagnosis of Ventilator associated Pneumonia. Chest. 2000;117:195-97

4. Dorca J, Manresa F, Esteban L. Efficacy, safety, and therapeutic relevance of transthoracic aspiration with ultrathin needle in non ventilated nosocomial pneumonia. Am J Respir Crit Care Med. 1995;151:1491-96.

5. El-Ebiary M, Torres A, González J, Puig de la Bellacasa J, García C, Jiménez de Anta MT, et al. Quantitative cultures of endotracheal aspirates for the diagnosis of ventilator-associated pneumonia. Am Rev Respir Dis 1993;148:1552-7.

6. Salata RA, Lederman MM, Shales DM, Jacobs MR, Eckstein E,Tweardy JJ. Diagnosis of nosocomial pneumonia in intubated, intensive care unit patients. Am Rev Respir Dis 1987;135:426-32.

7. McCauley LM, Webb BJ, Sorensen J, Dean NC. Use of Tracheal Aspirate Culture in Newly Intubated Patients with Community-Onset Pneumonia. Ann Am Thorac Soc. 2016;13(3):376-81.

8. Slagle TA, Bifano EM, Wolf JW, Gross SJ. Routine endotracheal cultures for the prediction of sepsis in ventilated babies. Arch Dis Child. 1989;64(1):34-8.

9. Valencia Arango M, Torres Martí A, Insausti Ordeñana J, Alvarez Lerma F, Carrasco Joaquinet N, Herranz Casado M, Tirapu León JP; Diagnostic value of quantitative cultures of endotracheal aspirate in ventilator-associated pneumonia: a multicenter study.Arch BroncoPneumol. 2003;39(9):394-9.

10. AK Shah, S Nandi, P Dhar. Prevalence of the bacterial isolates in the endotracheal tube according to the culture and sensitivity in patients of ICU- a tertiary medical college and hospital, Kolkata, West Bengal. International journal of contemporary medical research. 2016;3(6): 1775 .

11. Jakribettu RP, Boloor R. Characterisation of aerobic bacteria isolated from endotracheal aspirate in adult patients suspected ventilator associated pneumonia in a tertiary care center in Mangalore. Saudi Journal of Anaesthesia. 2012;6(2):115-9.

12. Ahmed NH, Hussain T, Biswal I. Antimicrobial resistance of bacterial isolates from respiratory secretions of ventilated patients in a multi-specialty hospital. Avicenna J Med. 2015;5(3):74-8.

13. Roopa C, Gangane Ravindranath. Pathogens Causing Ventilator Associated Pneumonia and Their Antibiogram in a Tertiary Care Hospital in North Karnataka MRIMS J Health Sciences 2015;3(1):11-15.

14. Ankita Patel, Sucheta Lakhani, Radhika Khara. Microbiological profile of Ventilator associated pneumonia at ICU of rural based teaching hospital. Int J Biol Med Res.2015;6(1):4732-6.

15. Fujitani and Yu. Quantitative Cultures for Diagnosing VentilatorAssociated Pneumonia: A Critique. Clin. Infect. Dis. 2006;43:S106-13.

16. T Rajasekhar, K Anuradha, T Suhasini, V Lakshmi. . The role of quantitative cultures of non-bronchoscopic samples in ventilator associated pneumonia. Indian J. Med. Microbiol.. 2006;24(2):107-13

17. Baselski VS, el-Torky M, Coalson JJ, Griffin JP. The standardization of criteria for processing and interpreting laboratory specimens in patients with suspected ventilatorassociated pneumonia. Chest 1992; 102:571-9.

18. www.icmr.nic.in/Publications/SOP/SOP_Bacteriology.pd f. downloaded on 14/11/2017

19. CLSI. Performance Standards for Antimicrobial Susceptibility Testing. 27th ed. CLSI supplement M100.Wayne, PA: Clinical and Laboratory Standards Institute; 2017.

20. Joseph NM, Sistla S, Dutta TK,Badhe AS, Rasitha D, Parija SC. Role of semi-quantitative and quantitative cultures of endotracheal aspirates in the diagnosis of ventilator-associated pneumonia. AMJ. 2010;3(10): 627632

21. Dey A and Bairy I. Incidence of multidrug resistant organisms causing ventilator associated pneumonia in a tertiary care hospital: A nine months prospective study. Ann Thorac Med. 2007;2(2):52-7.

22. Hamet M, Pavon A, Dalle F, PrinS,Pechinot A, Quenot J, Charles P .Candida sp. airway colonization couldpromote antibiotic-resistant bacteriaselection in the patients with suspected ventilator-associated pneumonia.Intensive Care Med. 2012;38. 
23. Trouillet, J.L., et al., Ventilator-associated pneumonia caused bypotentially drug-resistant bacteria. Am J RespirCrit Care Med, 1998;157(2):531-9.

24. Rodríguez-Baño J, Pascual A. Clinical significance of extended-spectrumbeta-lactamases. Expert Rev Anti Infect Ther. 2008 Oct;6(5):671-83.

25. Goel et al. Ventilator-associated pneumonia in medical intensive careunit: Microbial etiology, susceptibility patterns of isolated organism andoutcome. Indian $\mathrm{J}$ Anaesth 2012;56:558-62.

26. Veenakrishnamurthy, Vijay Kumar GS et al.,"Ventilator associatedpneumonia: bacterial isolates and its antibiotic resistance pattern.'Int J Biol Med Res.2013 4(2): 31353138

27. https://www.cdc.gov/nhsn/pdfs/training/2017/Gross_Mar ch21.pdf 